\title{
Erratum: "Methods for Calculating the Spectral Properties of Multilayer Anisotropic Structures Based on Crossed Polarized Gratings" [Technical Physics 46 (12), 1489 (2001)]
}

A. V. Arzhannikov and S. A. Kuznetsov

e-mail: sakuzn@inp.nsk.su

Page 1389, left column:

row 10 from below should read as "Conventional multiple-beam microwave interferometers ..." 